American Journal of Pharmaceutical Education 2018; 82 (9) Article 7010.

\title{
COMMENTARY
}

\section{A Modified Approach to Setting Curriculum Boundaries in Pharmacy School}

\author{
Daniel S. Longyhore, PharmD, MSEd, ${ }^{a}$ Dave L. Dixon, PharmD, ${ }^{b}$ Zachary R. Noel, PharmD ${ }^{c}$ \\ ${ }^{a}$ Nesbitt School of Pharmacy, Wilkes University, Wilkes-Barre, Pennsylvania \\ ${ }^{\mathrm{b}}$ Virginia Commonwealth University School of Pharmacy, Richmond, Virginia \\ ${ }^{c}$ University of Maryland School of Pharmacy, Baltimore, Maryland \\ Submitted February 9, 2018; accepted May 31, 2018; published November 2018.
}

\begin{abstract}
Doctor of Pharmacy department heads are responsible for determining the breadth and depth of content within courses. While the Accreditation Council for Pharmacy Education (ACPE) provides standards for what content, skills, and abilities should be included in PharmD education, the process that schools and colleges use to determine the degree to which these measured outcomes are taught is variable. As new topics and content for instruction are identified, schools and colleges are faced with either extending the PharmD curriculum length, removing other content, or diminishing the depth that other content is covered to make room for new content. To assist with these decisions, the Ebel grid is a tool that can be used to identify the criticality and relevance of encountered topics as well as guide preAPPE curriculum selection.
\end{abstract}

Keywords: curriculum inclusion, Ebel method

Imagine you are in a curricular-development committee meeting focused on identifying areas of the curriculum that are missing or insufficiently represented. A faculty member shares feedback that advanced pharmacy practice experience (APPE) students lack the basic knowledge and skills for interpreting electrocardiograms (EKG) and drug-induced EKG changes. While committee members agree that it would be ideal to include basic EKG instruction in the curriculum, identifying the extent to which the topic should be taught and what areas of the curriculum will be removed or diminished to make room for it is far more polarizing.

Although this is an isolated illustration, the dilemma of determining what to include in the pre-APPE curriculum is far from an isolated problem. The method for choosing specific knowledge, skills, values and abilities in pharmacy education is not clearly defined. Although the ACPE Standards 2016 offers required elements for the Doctor of Pharmacy curriculum, these recommendations are overarching and do not prescribe exact pharmacotherapeutic topics which must be included. ${ }^{1}$ For example, regarding content, Appendix A requires inclusion of pharmacotherapeutics, but does not place preference to organ system (eg, cardiovascular) or diseases/ conditions (eg, arrhythmias). For pharmacy curriculum content guidance, schools and colleges may turn to the

Corresponding Author: Zachary R. Noel, University of Maryland School of Pharmacy, 20 N. Pine St., Baltimore, MD 21201. Tel: 410-706-5819. E-mail: znoel@rx.umaryland.edu
2016 American College of Clinical Pharmacy (ACCP) "Pharmacotherapy Didactic Curriculum Toolkit." ${ }^{2}$ This guidance document places tiers of importance ranging from $I=$ most important to $I I I=$ least important, with I recommending students be, "proficient in providing care to patients with the disease." For major topics, such as cardiac arrhythmias, the toolkit recommends inclusion to the highest level of student knowledge. However, within the topic of cardiac arrhythmias, the depth and breadth of instruction (eg, EKGs) is not specified. Both the Center for the Advancement of Pharmacy Education (CAPE) outcomes and the new Entrustable Professional Activities (EPA) document offer the same level of guidance, describing the general abilities required of all pharmacy graduates, but not prescribing or giving preference to specifics within each ablity.,

These unknowns can make designing and executing a curriculum challenging. As the scope of pharmacy and required practice skills expand, along with the ever-increasing advances in the treatment and understanding of diseases, schools and colleges have three options: extend the length of the professional curriculum, attempt to address the knowledge and ability outcomes to a lesser depth, or remove specific practice skills and abilities from the curriculum. This will certainly lead to inequities in the depth and breadth of graduating pharmacy students' abilities from one curriculum to the next, but this is already happening in schools and colleges across the United States. As an example, a 2017 survey of complementary and alternative medicine education in Doctor of Pharmacy 


\section{American Journal of Pharmaceutical Education 2018; 82 (9) Article 7010.}

programs, which is an ACPE required element, showed a vast difference in how each manages this topic. ${ }^{5}$ The majority of programs offered two to three hours of instruction per week on complementary and alternative medicine, yet not one topic was consistently covered in every single course. Most often, dietary/nutritional supplements and herbal medicine were included, but as few as 15 of the 190 reported courses included topics such as spiritual healing or biofeedback practices. This leads to the question: what criteria are used for topic inclusion? And, a question even broader to pharmacy education: how do curriculum development teams choose the abilities that meet the required elements of a pharmacy graduate? Is it a systematic process or instructor dependent?

In 1971, author Sidney Drumheller published the Handbook of Curriculum Design for Individualized Instruction. ${ }^{6}$ Drumheller discusses categorizing aspects of a curriculum as "need to know" and "nice to know," with the former being required elements and the latter being optional. Within health education, Cordingly and colleagues published their experience with curriculum development for including topics in psychology in medical education. ${ }^{7}$ Using an assembly of medical professionals and a systematic process, a working group was able to place topics into one of three categories: core knowledge, professional practice, and educational process. In terms of the pharmacy education curriculum, this may translate into what should be taught and assessed in the classroom (pre-APPE need to know), what could be taught and assessed as part of the curriculum (pre-APPE nice to know), and what should be reserved for advanced experiential education (as needed on APPE).

To actualize the aforementioned process and determine how to classify knowledge, skills, values, and abilities, consider the work of Robert Ebel and his methods for standard setting in examination performance. To identify standards, Ebel created a grid to plot criticality versus relevance. ${ }^{8}$ In pharmacy education, "criticality" may refer to the importance of the topic and "relevance" to the frequency with which a topic is encountered. As an example, consider a patient presenting a prescription to a pharmacist for levofloxacin. While performing the final verification, the pharmacist is alerted to a potential interaction with the patient's methadone. What reaction should the pharmacist have to this interaction? How should he or she educate the patient about the interaction? What should be stated or recommended to the prescriber? Using the modified Ebel grid in Figure 1, a curriculum may map the ability to manage this drug interaction as "need to know." The criticality of the ability is "high" because combining these medications may lead to Torsades de pointes, a potentially fatal arrhythmia. Also, it is

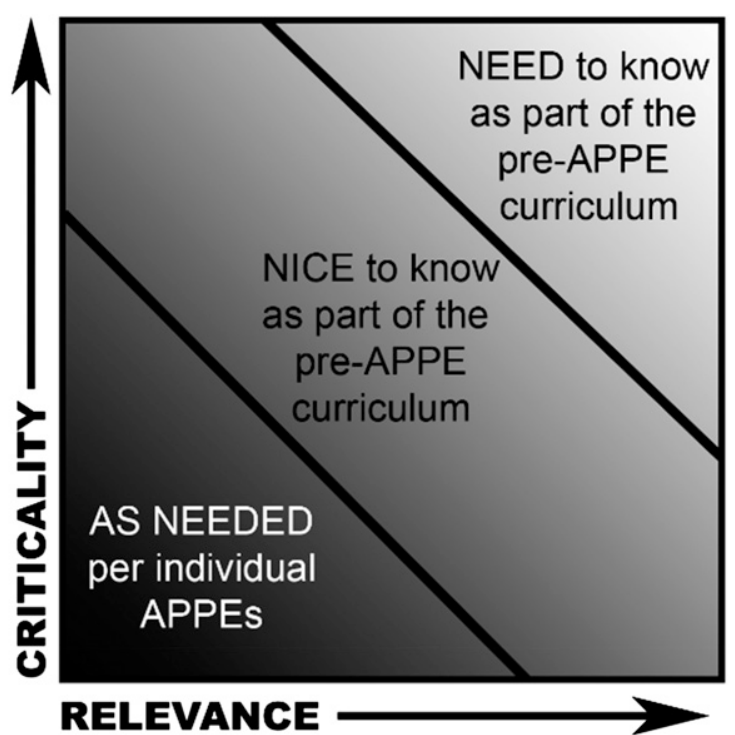

Relevance: The frequency with which a general pharmacy practitioner will encounter the need to use the knowldge, skill, or ability.

Criticality: The importance of possessing the knowledge, skill, or ability as a general pharmacy practitioner.

Figure 1. Modified Ebel Grid for Setting Curriculum Boundaries in Pharmacy School.

of "high" relevance because interactions with other QT prolonging medications are among the most common interaction alerts pharmacists encounter. ${ }^{9}$ Although most of these interactions do not require intervention, this does not undermine the importance of critically assessing and evaluating the interaction to determine whether action needs to be taken.

On the contrary, consider a patient who presents a prescription for dofetilide. The pharmacist, upon reviewing the patient's medication profile, calls the electrophysiologist to verify that the QTc is not greater than $500 \mathrm{msec}$. From a criticality perspective, interpreting the QTc interval is "high." However, in general pharmacy practice, this type of interaction may be relatively rare, thereby being of "low" relevance. Returning to Figure 1, this would be considered "nice to know" on the modified Ebel grid. It is, however, important to remember that the modified Ebel grid is relative. In this scenario, interpreting the QTc interval is considered "nice to know" for a general pharmacist practitioner; however, for an inpatient pharmacist routinely involved in the care of patients being initiated on dofetilide, both the criticality and the relevance would be considered "high" and "need to know." Similarly, for the pharmacist working as an ambulatory care pharmacist in a diabetes clinic, knowing the exact QTc interval for someone on dofetilide is likely "low" for both relevance and criticality. Returning the framework to 


\section{American Journal of Pharmaceutical Education 2018; 82 (9) Article 7010.}

the pre-APPE curriculum in pharmacy education, the target for the curriculum should be to that of a general pharmacist practitioner. As students progress to their APPEs and post-graduate training, the frame may shift and/or become more focused.

The decision to include or exclude specific knowledge, skills, and values from the pre-APPE curriculum lies, in part, with the ACPE standards; however, the depth to which those abilities are covered lie with the faculty of the school or college. As decisions are made, consider the "need to know" abilities for students entering their APPE year. The decision should be informed by faculty and/or a curriculum committee that has identified diverse practice areas. As faculty and committees review course syllabi, map content areas, and ensure students are meeting abilities requirements, they should be encouraged to vet the content through a modified Ebel grid to ensure the breadth and depth of instruction is acceptable for pharmacy students entering general pharmacy practice.

\section{REFERENCES}

1. Accreditation Council for Pharmacy Education. Accreditation standards and key elements for the professional program in pharmacy leading to the doctor of pharmacy degree. Standards 2016. https:// www.acpe-accredit.org/pdf/Standards2016FINAL.pdf.

2. Schwinghammer T, Crannage A, Boyce EG, et al. The 2016 ACCP Pharmacotherapy Didactic Curriculum Toolkit. Pharmacotherapy. 2016;36(11):e189-e194.

3. Medina MS, Plaza CM, Stowe CD, et al. Center for the Advancement of Pharmacy Education 2013 educational outcomes. Am J Pharm Educ. 2013;77(8):Article 162.

4. Haines ST, Pittenger AL, Stolte SK, et al. Core entrustable professional activities for new pharmacy graduates. Am J Pharm Educ. 2017;81(1):Article S2.

5. Scaletta A, Ghelani N, Sunny S. Complementary and alternative medicine education in U.S. schools and colleges of pharmacy. Curr Pharm Teach Learn. 2017;9(4):521-527.

6. Drumheller SJ. Handbook of Curriculum Design for Individualized Instruction: A Systems Approach [e-book]. 1971. Ipswich, MA: ERIC; 2018.

7. Cordingley L, Peters S, Hart J, et al. What psychology do medical students need to know? An evidence based approach to curriculum development. Health Soc Care Educ. 2013;2(2):38.

8. Ebel's Method, ExamDeveloper Help System, Pearson Vue ExamDeveloper. http://examdevhelp.pearsonvue.com/display/ EDHelp/Ebel\%27s + Method. Accessed November 11, 2017. 9. Van der Sijs H, Kowlesar R, Klootwijk A, Nelwan S, Vulto A, van Gelder T. Clinically relevant QTc prolongation due to overridden drug-drug interaction alerts: a retrospective cohort study. Br J Clin Pharmacol. 2009;67(3):347-354. 